\title{
Efeito do Período de Chuva no Controle de Euphorbia heterophylla E Ipomoea purpurea PELOS HERBICIDAS GLYPHOSATE E SUlFosate ${ }^{1}$
}

\author{
Effect of Rainfall on Euphorbia heterophylla and Ipomoea purpurea control by Glyphosate and \\ Sulfosate
}

MONQUERO, P.A. ${ }^{2}$ e SILVA, A.C. ${ }^{3}$

\begin{abstract}
RESUMO - Avaliou-se a influência do período de chuva sobre a eficácia dos herbicidas sulfosate e glyphosate no controle de Euphorbia heterophylla e Ipomoea purpurea. O experimento foi instalado em casa de vegetação, no Centro de Ciências Agrárias/UFSCar, em Araras, SP. Os tratamentos, além da testemunha, consistiram na aplicação de duas doses em equivalente ácido dos herbicidas sulfosate $\left(0,66\right.$ e $\left.1,32 \mathrm{~kg} \mathrm{ha}^{-1}\right)$ e glyphosate $(0,72$ e $1,44 \mathrm{~kg} \mathrm{ha}^{-1}$ ), sendo as plantas tratadas submetidas à simulação de chuva de $20 \mathrm{~mm}$ durante 30 minutos, nos intervalos de 2, 4, 6, 8 e 48 horas após a aplicação. O delineamento experimental utilizado foi o inteiramente casualizado, com quatro repetições. A eficácia no controle das plantas daninhas foi avaliada 7, 14 e 21 dias após a aplicação dos tratamentos (DAT) e a matéria seca, aos 28 DAT. E. heterophylla não foi eficazmente controlada pelo herbicida glyphosate, exigindo intervalo sem chuva superior a 24 horas após a aplicação para obter, na maior dose, controle de apenas 60\%. Por sua vez, o herbicida sulfosate proporcionou controle de $75 \%$, mesmo na menor dose, quando a chuva ocorreu quatro horas depois da aplicação. I. purpurea também não foi controlada de maneira eficaz pelo herbicida glyphosate, independentemente da dose e do intervalo de chuva; no entanto, somente a maior dose do herbicida sulfosate foi eficiente no controle desta espécie, a partir de quatro horas sem chuva. Para ambas as espécies, o herbicida sulfosate foi menos afetado pelo período da chuva.
\end{abstract}

Palavras-chave: eficácia, precipitação, toxicidade.

\begin{abstract}
The influence of rainfall occurrence was evaluated on the efficacy of the herbicides glyphosate and sulfosate applied in post-emergence on Euphorbia heterophylla and Ipomoea purpurea. The experiment was conducted under greenhouse conditions at the Centro de Ciências Agrárias/UFSCar, in Araras-SP, Brazil. The treatments, plus control, consisted of two doses, in acid equivalent of sulfosate (0.66 and $\left.1.32 \mathrm{~kg} \mathrm{ha}^{-1}\right)$ and glyphosate $(0.72$ and $1.44 \mathrm{~kg} \mathrm{ha-1)}$ submitted to rainfall simulation of $20 \mathrm{~mm}$ for 30 minutes, 2, 4, 6, 8, and 48 h after application. The experimental design was completely randomized with four replicates. Weed control efficacy was evaluated 7, 14, and $21 \mathrm{~d}$ after treatments (DAT) and dry biomass was evaluated 28 DAT. $\boldsymbol{E}$. heterophylla was not efficiently controlled by glyphosate, demanding rainfall interval longer than $24 \mathrm{~h}$ after application to attain a moderate control of $60 \%$ when the highest rate was applied. On the other hand, sulfosate provided control of 75\% when rainfall occurred just $4 \mathrm{~h}$ after application. I. purpurea was not controlled by glyphosate, either Sulfosate was only effective in controlling this species at the highest application rate, without rainfall. For both species, sulfosate was less affected by rainfall occurrence.
\end{abstract}

Keywords: efficacy, rainfall, toxicity.

Recebido para publicação em 11.9.2006 e na forma revisada em 17.4.2007.

Professora Adjunta do Dep. de Recursos Naturais e Proteção Ambiental/Centro de Ciências Agrárias/UFSCar <pamonque@cca.ufscar.br> Rodovia Anhanguera, km 174 - Araras-SP; ${ }^{3}$ Pesquisadora científica da Agência Paulista de Tecnologia dos Agronegócios - APTA - Pólo Regional da Alta Sorocabana <andreiacs@aptaregional.sp.gov.br>. 


\section{INTRODUÇÃO}

Euphorbia heterophylla é uma planta daninha comum nas regiões Sul, Sudeste e CentroOeste do Brasil. Em áreas produtoras de soja dos Estados do Paraná e Rio Grande do Sul, relatou-se resistência de E. heterophylla a herbicidas inibidores de enzima acetolactato sintase - ALS, que são os mais utilizados (Gazziero et al., 1998; Vidal \& Merotto Jr., 1999). Assim, a utilização de herbicidas com mecanismos de ação diferentes dos inibidores da ALS, como o glyphosate, é uma estratégia importante no manejo da resistência.

Ipomoea purpurea, popularmente conhecida como corda-de-viola, também é uma espécie daninha altamente prejudicial, especialmente em culturas anuais, pelo fato de seu ciclo ser mais longo que o da cultura, além de os ramos se entrelaçarem nas plantas, dificultando a colheita (Kissmann \& Groth, 1999).

Essas duas espécies daninhas são controladas pelos herbicidas glyphosate e sulfosate, que são os principais herbicidas usados como dessecante em plantio direto (Rodrigues \& Almeida, 2005). Nas formulações de glyphosate podem ser utilizados os sais de potássio, de isopropilamina e amônio, enquanto na formulação de sulfosate utiliza-se o sal trimetilsulfônico. Esses herbicidas não são seletivos e apresentam ação sistêmica, sendo usados em pós-emergência no controle de plantas daninhas anuais e perenes. Várias revisões descrevem como único mecanismo de ação a inibição da enzima 5-enolpiruvilshiquimato fosfato sintetase (EPSPs). Como conseqüência dessa inibição, não ocorre a sintese dos aminoácidos aromáticos fenilalanina, tirosina e triptofano e de compostos secundários, como alcalóides, coumarinas e flavonóides (Amrhein et al., 1980).

A absorção do glyphosate e sulfosate ocorre por um processo bifásico, que envolve rápida penetração inicial através da cutícula, seguido por absorção simplástica lenta. A duração desse processo depende de vários fatores, como a espécie e a idade da planta, as condições ambientais, a concentração do herbicida na calda e do surfatante (Ruitner \& Meinen, 1998). Após a penetração do herbicida na planta, é necessário que ocorra a translocação simplástica, através de tecidos vasculares, para os sítios- alvo (Satichivi et al., 2000). O movimento do glyphosate pelo floema segue a mesma rota dos produtos da fotossintese (açúcares), ocorrendo das folhas fotossinteticamente ativas em direção às partes das plantas que utilizam esses açúcares para crescimento, manutenção, metabolismo ou armazenamento, como, por exemplo, raizes, tubérculos, rizomas, folhas jovens e zonas meristemáticas (Dellacioppa et al., 1986). A quantidade de açúcar translocada para cada uma dessas partes se altera durante o ciclo de vida da planta e, conseqüentemente, influencia o movimento do herbicida. Portanto, condições que favoreçam a fotossíntese auxiliam também a translocação desses herbicidas (Dellacioppa et al., 1986).

As precipitações pluviais após a aplicação do herbicida podem comprometer o seu desempenho em pós-emergência. Assim, o intervalo entre a aplicação e a ocorrência de chuvas, bem como a quantidade e a intensidade destas, além dos diferentes herbicidas e tipos de formulações, influenciam a eficiência de controle de plantas daninhas (Anderson \& Arnold, 1984). O glyphosate é lentamente absorvido pela cutícula, podendo ser necessário um período de seis horas sem chuvas após a aplicação para haver controle satisfatório de plantas suscetiveis. Para diminuir o intervalo temporal entre a aplicação e a ocorrência de chuvas, são adicionados surfatantes ao produto, visando conferir maior afinidade da molécula aos lipídios, facilitando, assim, a absorção foliar (Rodrigues \& Almeida, 2005).

Pedrinho Junior et al. (2002) estudaram a influência do momento da chuva após a aplicação do herbicida glyphosate isolado e em mistura com adjuvantes (uréia, sulfato de amônio e óleo vegetal) na dessecação de plantas daninhas. A simulação de chuvas em um, dois, quatro ou seis horas após a aplicação do glyphosate foi prejudicial à ação do herbicida, porém a adição de uréia foi uma boa alternativa para o controle de plantas daninhas, principalmente no verão, quando há maior incidência de chuvas.

Foi observado que a ocorrência de orvalho após a pulverização de glyphosate prejudicou a eficiência de controle de Brachiaria decumbens, contudo, o tempo dessa ocorrência foi determinante, sendo necessárias oito horas 
sem orvalho para se obter controle maior que 80\% dessa espécie (Santos et al., 2004).

O objetivo deste trabalho foi avaliar a influência da chuva, simulada em diferentes intervalos após a aplicação, na eficácia dos herbicidas glyphosate (Roundup Original) e sulfosate (Touchdown) sobre as plantas daninhas E. heterophylla e I. purpurea.

\section{MATERIAL E MÉTODOS}

O experimento foi instalado em casa de vegetação do Centro de Ciências Agrárias da UFSCar, Araras-SP. As plantas daninhas $I$. purpurea e E. heterophylla foram semeadas em vasos com capacidade para 0,3 L, utilizando como substrato uma mistura de terra (Latossolo Vermelho eutrófico) e matéria orgânica (2:1), mantida com umidade próxima à capacidade de campo. As características químicas do substrato são apresentadas na Tabela 1 .

O delineamento utilizado foi o inteiramente casualizado, com quatro repetições. Após a emergência, as plântulas foram desbastadas para obtenção de três plantas por vaso. Os tratamentos consistiram na aplicação de duas doses, em equivalente ácido, de sulfosate $(0,66$ e 1,32 $\mathrm{kg} \mathrm{ha}^{-1}$ ) e glyphosate $\left(0,72\right.$ e 1,44 $\left.\mathrm{kg} \mathrm{ha}^{-1}\right)$, que correspondem a 2 e $4 \mathrm{~L} \mathrm{ha}^{-1}$ dos respectivos produtos comerciais, e seis períodos sem chuva após as aplicações dos herbicidas $(2,4,6$, 8,24 e 48 h), além dos tratamentos testemunhas, sem os herbicidas.

Os herbicidas foram aplicados quando as plantas daninhas apresentavam o terceiro par de folhas verdadeiras, utilizando-se pulverizador costal pressurizado por $\mathrm{CO}_{2}$, à pressão constante de 2,5 $\mathrm{kgf} \mathrm{cm}^{2}$, barra de aplicação provida de bicos com pontas de pulverização do tipo leque, com jato plano, modelo XR 80.02, e consumo de calda de $200 \mathrm{~L} \mathrm{ha}^{-1}$. As aplicações foram feitas fora da casa de vegetação, iniciando-se às 9 horas, com umidade relativa de $65 \pm 4 \%$ e temperatura do ar de $28 \pm 3{ }^{\circ} \mathrm{C}$ até o final das aplicações. A chuva de $20 \mathrm{~mm}$ foi simulada com um sistema de irrigação por aspersão.

Aos 7, 14 e 21 dias após a aplicação dos tratamentos (DAT) foram realizadas avaliações visuais da eficácia de controle, utilizando-se escala de notas em que $0 \%$ representa ausência de sintomas de intoxicação e $100 \%$, morte completa das plantas. Aos 21 DAT, as plantas foram cortadas rente ao solo e secas em estufa de ventilação forçada a $60 \pm 2{ }^{\circ} \mathrm{C}$, para quantificação da matéria seca aos 28 DAT.

Os resultados de controle visual e matéria seca foram submetidos à análise de variância, e, quando significativos $(\mathrm{p}<0,05)$, foram ajustadas curvas de regressão.

\section{RESULTADOS E DISCUSSÃO}

Na Figura 1, observa-se o controle visual das espécies daninhas pelos herbicidas glyphosate e sulfosate, submetidos a diferentes intervalos de chuva após a aplicação. E. heterophylla foi mais suscetivel aos herbicidas em relação a I. purpurea, nas menores doses, independentemente do intervalo de chuva. A espécie $I$. purpurea somente foi efetivamente controlada na maior dose do sulfosate.

De modo geral, não foi observada diferença entre a maior e a menor dose do sulfosate no controle de $E$. heterophylla, especialmente na avaliação feita aos 21 DAT, verificando-se controle em torno de $100 \%$. Desse modo, a menor dose pode ser utilizada no controle da espécie com resultado satisfatório. O glyphosate proporcionou controle máximo das espécies na maior dose - em torno de 60\%, ligeiramente superior à menor dose.

A chuva após a aplicação apresentou impacto negativo na eficácia dos herbicidas, havendo melhor controle das espécies com o aumento do intervalo entre a aplicação dos herbicidas e a chuva simulada. O herbicida

Tabela 1 - Características químicas do substrato

\begin{tabular}{|c|c|c|c|c|c|c|c|c|c|c|}
\hline $\mathrm{pH} \mathrm{CaCl}{ }_{2}$ & $\mathrm{MO}$ & $\mathrm{P}$ & $\mathrm{S}-\mathrm{SO}_{4}$ & $\mathrm{~K}$ & $\mathrm{Ca}$ & $\mathrm{Mg}$ & $\mathrm{Al}$ & SB & $\mathrm{T}$ & V\% \\
\hline & $--\mathrm{g} \mathrm{dm}^{-3}--$ & \multicolumn{2}{|c|}{----- $\mathrm{mg} \mathrm{dm}^{-3}$---- } & \multicolumn{4}{|c|}{------------- $\mathrm{mmol}_{\mathrm{c}} \mathrm{dm}^{-3}$------------- } & & & \\
\hline 6,4 & 47 & 468 & 127 & 15.2 & 111 & 29 & 0 & 155,2 & 170,2 & 91 \\
\hline
\end{tabular}




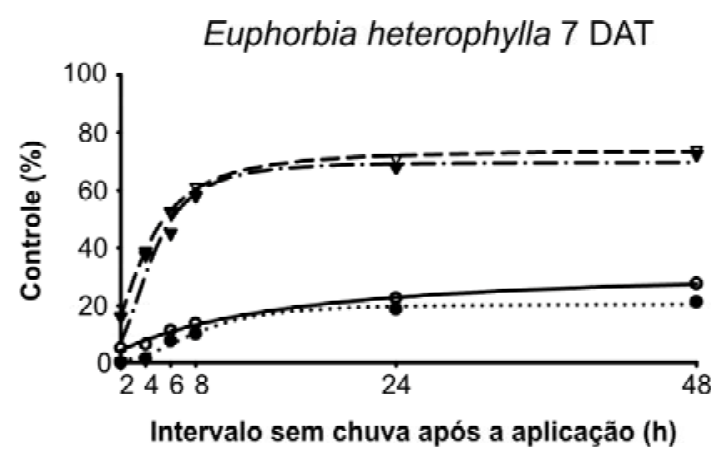

....... Gly $\left(2 \mathrm{~L} \mathrm{haA}^{-1}\right)\left(\hat{\mathrm{Y}}=20,5227 /\left(1+\left(x / 7,9717^{2 \text { 20556 }}\right)\right) \mathrm{R}^{2}=0,987\right.$

- Gly $\left(4 \mathrm{Lha} \mathrm{A}^{-1}\right)\left(\hat{\mathrm{Y}}=33,3080 /\left(1+(x / 11,8001)^{-1,0917}\right)\right) R^{2}=0,991$

$\rightarrow$. Sul $\left(2 \mathrm{~L} \mathrm{ha} a^{-1}\right)\left(\hat{Y}=69,8307 /\left(1+(x / 4,3689)^{27711}\right)\right) \quad R^{2}=0,959$

- $\nabla-\operatorname{Sul}\left(4 \mathrm{Lha}^{-1}\right)\left(\hat{\mathrm{Y}}=73,7721 /\left(1+(\mathrm{x} / 3,7791)^{-2,0105}\right)\right) R^{2}=0,999$

\section{Euphorbia heterophylla 14 DAT}

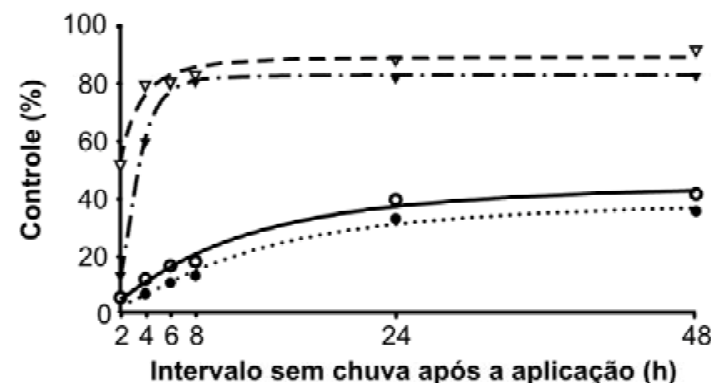

...*.. Gly $\left(2 \mathrm{~L} \mathrm{ha}{ }^{-1}\right)\left(\hat{\mathrm{Y}}=39,4829 /\left(1+\left(\mathrm{x} / 11,3626={ }^{-1.6482}\right)\right) \mathrm{R}^{2}=0,982\right.$

- $\operatorname{Gly}\left(4 \mathrm{Lha}^{-1}\right)\left(\hat{\mathrm{Y}}=49,4939 /\left(1+\left(\mathrm{x} / 9,6691=^{-1,452}\right)\right) R^{2}=0,986\right.$

$\rightarrow$ Sul $\left(2 \mathrm{~L} \mathrm{ha}^{-1}\right)\left(\hat{\mathrm{Y}}=82,1955 /\left(1+\left(\mathrm{x} / 3,1166={ }^{-4.0106}\right)\right) \mathrm{R}^{2}=0,998\right.$

- $\nabla-\operatorname{Sul}\left(4 \mathrm{Lha}{ }^{-1}\right)\left(\hat{Y}=88,8684 /\left(1+\left(x / 1,6812={ }^{-1,9919}\right)\right) R^{2}=0,968\right.$

\section{Euphorbia heterophylla 21 DAT}

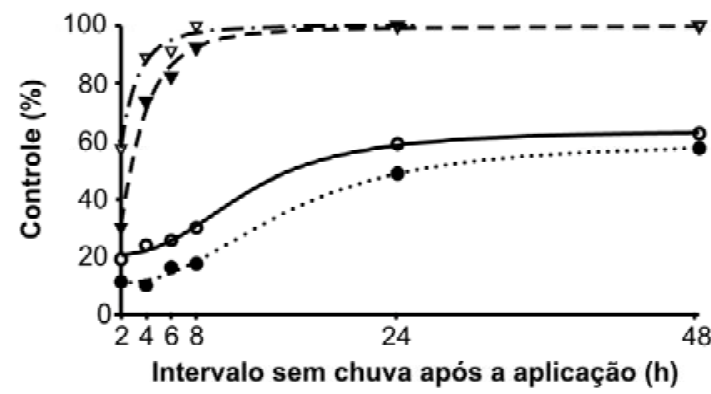

...*.. Gly $\left.\left(2 \mathrm{~L} \mathrm{ha}^{-1}\right)\left(\hat{\mathrm{Y}}=10,3478+\left(49,3701 /\left(1+(\mathrm{x} / 14,9989)^{-2.6034}\right)\right)\right)\right) \mathrm{R}^{2}=0,996$

- $\operatorname{Gly}\left(4 \mathrm{~L}\right.$ ha $\left.\left.{ }^{-1}\right)\left(\hat{\mathrm{Y}}=20,1509+\left(43,4384 /\left(1+(\mathrm{x} / 11,8962)^{-2.8924}\right)\right)\right)\right) R^{2}=0,997$

$\rightarrow$ Sul $\left(2 \mathrm{~L} \mathrm{ha}^{-1}\right)\left(\hat{\mathrm{Y}}=99,6769 /\left(1+(\mathrm{x} / 2,7746)^{-2,4121}\right)\right) \mathrm{R}^{2}=0,992$

- $\nabla$ - Sul $\left(4 \mathrm{~L}\right.$ ha $\left.^{-1}\right)\left(\hat{Y}=100,1868 /\left(1+(x / 1,7577)^{-2.3562}\right)\right) R^{2}=0,983$

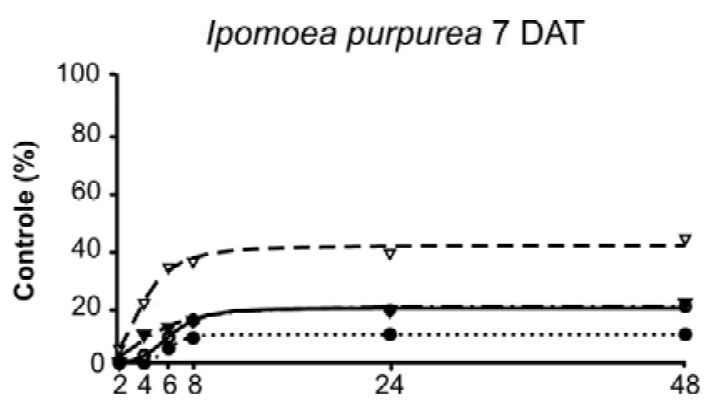

Intervalo sem chuva após a aplicação (h)

...... Gly $\left(2 \mathrm{~L} \mathrm{ha}^{-1}\right)\left(\hat{\mathrm{Y}}=9,9264 /\left(1+(\mathrm{x} / 6,0286)^{-7.0047}\right)\right) \quad \mathrm{R}^{2}=0,998$

- $\operatorname{Gly}\left(4 \mathrm{Lha}^{-1}\right)\left(\hat{\mathrm{Y}}=19,4179 /\left(1+(\mathrm{x} / 6,1609)^{-4.5513}\right)\right) \quad R^{2}=0,997$

$\rightarrow$ Sul $\left(2 \mathrm{Lha}^{-1}\right)\left(\hat{\mathrm{Y}}=20,0351 /\left(1+(\mathrm{x} / 4,6909)^{-2,4505}\right)\right) \quad R^{2}=0,953$

- $\nabla$ - Sul $\left(4 \mathrm{Lha}^{-1}\right)\left(\hat{\mathrm{Y}}=41,2193 /\left(1+(\mathrm{x} / 3,8705)^{-2.9819}\right)\right) \quad \mathrm{R}^{2}=0,985$

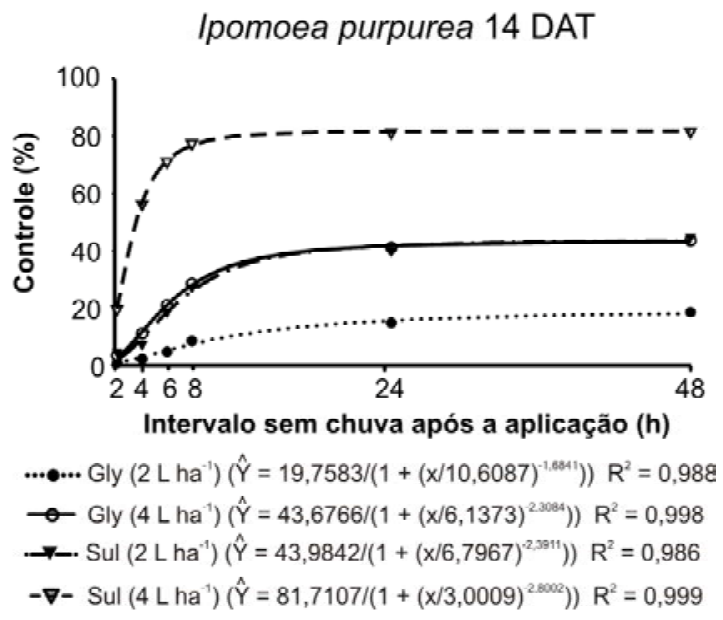

Ipomoea purpurea $21 \mathrm{DAT}$

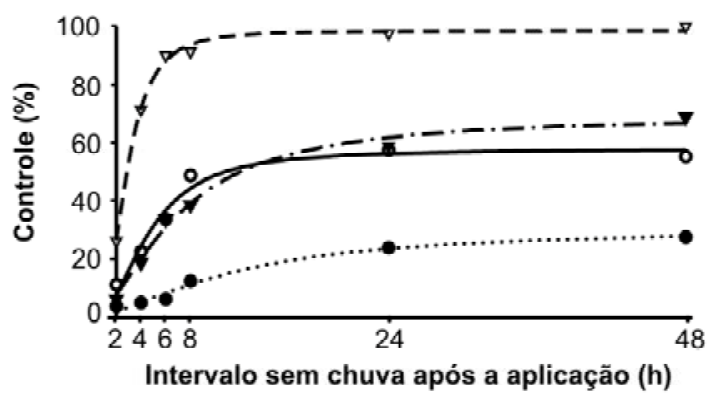

...*... Gly $\left(2\right.$ L ha $\left.^{-1}\right)\left(\hat{Y}=30,6063 /\left(1+(x / 11,2543)^{-1.5647}\right)\right) R^{2}=0,983$

- Gly $\left(4 \mathrm{Lha}^{-1}\right)\left(\hat{\mathrm{Y}}=57,812 /\left(1+(\mathrm{x} / 4,6244)^{21819}\right)\right) \mathrm{R}^{2}=0,974$

$\rightarrow$ Sul $\left(2 \mathrm{~L} \mathrm{ha}^{-1}\right)\left(\hat{\mathrm{Y}}=68,9888 /\left(1+(\mathrm{x} / 6,7990)^{-1,6025}\right)\right) R^{2}=0,990$

$-\nabla$ - Sul $\left(4 L_{h a}^{-1}\right)\left(\hat{Y}=98,4784 /\left(1+(x / 2,8448)^{-2.8656}\right)\right) R^{2}=0,997$

Figura 1 - Porcentagem de controle das plantas daninhas E. heterophylla e I. purpurea, avaliada aos 7, 14 e 21 dias após a aplicação dos tratamentos (DAT). 
Euphorbia heterophylla

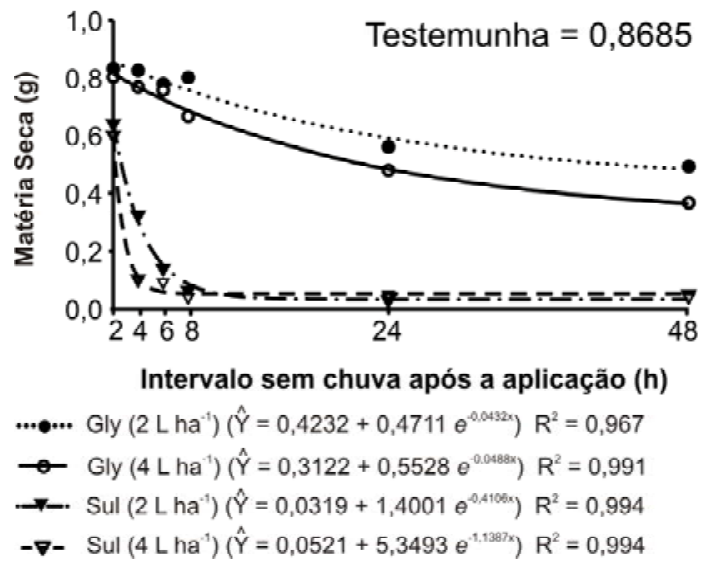

Ipomoea purpurea

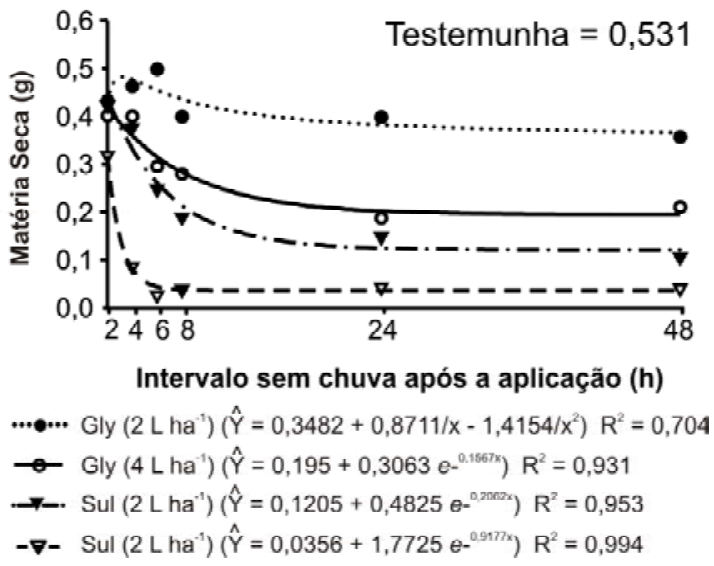

Figura 2 - Matéria seca de E. heterophylla e I. purpurea, avaliada aos 28 DAT, e testemunha sem herbicida.

sulfosate, na maior dose, proporcionou incremento acentuado no controle de I. purpurea entre os intervalos de quatro e seis horas, passando de 70 a $90 \%$ de controle, respectivamente. Para E. heterophylla, o controle foi de 90 para 93\% no mesmo período, evidenciando que a chuva após a aplicação do sulfosate obteve menor impacto nesta espécie.

O herbicida glyphosate apresentou maior suscetibilidade à chuva em relação ao sulfosate. A porcentagem de controle de E. heterophylla pelo glyphosate praticamente dobra quando se comparam 8 e 24 horas sem chuva, com tendência similar para as duas doses avaliadas, o que sugere perda do produto pela chuva. Por outro lado, para I. purpurea, não se observou alteração significativa na porcentagem de controle a partir de oito horas sem chuva, quando da utilização de glyphosate.

Segundo Bovey et al. (1990), a ocorrência de chuva quatro horas após a aplicação reduziu a ação do herbicida glyphosate no controle de Acacia farnesiana e Prosopis glandulosa.

Kirkwood \& Mckay (1994) salientam que a eficácia do glyphosate é dependente de processos como a retenção do herbicida na superfície foliar, a penetração foliar, a translocação na planta até o sítio de ação e inibição da enzima-alvo (EPSPs). Carvalho et al. (2003) demonstraram que esse herbicida nas doses de 0,72 e $0,96 \mathrm{~kg} \mathrm{ha}^{-1}$, aplicado isoladamente ou em mistura com chlorimuron-ethyl ou 2,4-D, foi eficaz na dessecação de E. heterophylla em sistema de plantio direto da cultura da soja.

Na Figura 2 são apresentados os dados de matéria seca das espécies, avaliadaaos 28 DAT, assim como da testemunha, sem herbicida. O glyphosate, na menor dose, proporcionou maior acúmulo de matéria seca das espécies; nos intervalos até oito horas com chuva, praticamente equivaleu-se à testemunha. De modo geral, para todos os herbicidas e doses estudadas, a matéria seca das plantas foi reduzida com o acréscimo no intervalo de tempo entre a aplicação e a simulação de chuva.

Este experimento permitiu concluir que a eficácia dos herbicidas foi acrescida à medida que se aumentou o intervalo entre a aplicação e a simulação de chuva; entretanto, o herbicida sulfosate foi menos afetado em todos os intervalos avaliados, em ambas as espécies estudadas. Além disso, a menor dose deste herbicida permitiu controle próximo a $100 \%$ de E. heterophylla, e a dose de 4,0 $\mathrm{Lha}^{-1}$ controlou as duas espécies.

\section{LITERATURA CITADA}

AMRHEIN, N. et al. The site of inhibition of the shikimate pathway by glyphosate. Plant Physiol., v. 66, p. 830-834, 1980.

ANDERSON, M. D.; ARNOLD, W. E. Weed control in sunflowers (Helianthus annuus) with desmediphan and phenmediphan. Weed Sci., v. 32, p. 310-314, 1984. 
BOVEY, R. W.; MEYER, R. E.; WHISENANT, S. G. Effect of simulated rainfall on herbicide performance in huisache (Acacia farnesiana) and honey mesquite (Prosopis glandulosa). Weed Technol., v. 4, p. 26-30, 1990.

CARVALHO, F. T. et al. Manejo químico das plantas daninhas Euphorbia heterophylla e Bidens pilosa em sistema de plantio direto da cultura de soja. Planta Daninha, v. 21, n. 1, p. 145-150, 2003.

DELLACIOPPA, G. N. et al. Translocation of the precursor of 5-enolpyruvylshikimate-3-phosphate synthase into chloroplasts of higher plants in vitro. Proc. Nat. Acad. Sci., v. 83 , p. $6873-6877,1986$.

GAZZIERO, D. L. P. et al. Resistência de amendoim-bravo aos herbicidas inibidores da enzima ALS. Planta Daninha, v. 16, n. 2, p. 117-125, 1998.

KIRKWOOD, R. C.; MCKAY, I. Accumulation and elimination of herbicides in select crop and weed species. Pestic. Sci., v. 42, p. 241-248, 1994.

KISSMANN, K. G.; GROTH, G. Plantas infestantes e nocivas. 2. ed. São Paulo: BASF, 1999. 978 p.
PEDRINHO JÙNIOR, A. F. F. et al. Influência da chuva na eficiência do glyphosate em mistura com adjuvantes na dessecação de plantas daninhas. Planta Daninha, v. 20, n. 2, p. 263-271, 2002.

RODRIGUES, B. N.; ALMEIDA, F. S. Guia de herbicidas. 5. ed. Londrina: IAPAR, 2005. 591 p.

RUITNER, H.; MEINEN, E. Influence of water stress and surfactant on the efficacy, absorption, and translocation of glyphosate. Weed Sci., v. 46, n. 3, p. 289-296, 1998.

SANTOS, J. L. et al. Influência do orvalho na eficiência do glyphosate sobre a Brachiaria decumbens. Planta Daninha, v. 22, n. 2, p. 285-291, 2004.

SATICHIVI, N. M. et al. Absorption and translocation of glyphosate isopropylamine and trimethysulfonium salts in Abutilon theophrasti and Setaria faberi. Weed Sci., v. 48, p. $675-679,2000$.

VIDAL, R. A.; MEROTTO JÚNIOR, A. Resistência de amendoim-bravo aos herbicidas inibidores da enzima acetolactato sintase. Planta Daninha, v. 17, n. 3, p. $367-$ 373, 1999.

WERLANG, R. C. et al. Efeitos da chuva na eficiência de formulações e doses de glyphosate no controle de Brachiaria decumbens. Planta Daninha, v. 21, n. 1, p. 121-130, 2003. 